\title{
HYPERGEOMETRIC FUNCTIONS ON COMPLEX MATRIX SPACE
}

\author{
KENNETH I. GROSS AND DONALD ST. P. RICHARDS
}

\section{INTRODUCTION}

In [6] we presented the general foundations for a theory of hypergeometric functions of matrix argument over real division algebras. In this note, we further develop the fine structure of these functions over the complex field, including series expansions, integral representations, asymptotics, differential equations, addition formulas, multiplication formulas, summation theorems, transformation properties, etc. Especially important in this paper are the operator-valued hypergeometric functions, required for (nonspherical) expansions such as addition formulas by the noncommutativity of matrix multiplication. These functions generalize the operator-valued Bessel functions studied in [5].

Hypergeometric functions of matrix argument arise naturally in applications ranging from multivariate statistics, quantum physics, and molecular chemistry, to harmonic analysis, group representations, and number theory. (See the references in [6].) These diverse applications amplify the need to develop the fine structure systematically and to the greatest extent possible.

We briefly review the definition of hypergeometric functions of matrix argument from [6]. Let $\mathbf{F}$ be the real field, the complex field, or the quaternions. Denote by $S$ the space of all $n \times n$ Hermitian matrices $s=s^{*}$ over $\mathbf{F}$, on which the group $G=G L(n, \mathbf{F})$ of invertible $n \times n$ matrices $g$ over $\mathbf{F}$ acts by $s \mapsto g^{*} s g$. Then $K=\left\{k \in G: k^{*} k=1\right\}$ is the isotropy subgroup of the identity matrix 1, the open cone $P$ in $S$ of positive-definite $n \times n$ matrices is the orbit under $G$ of 1 , and $P$ can be identified with the symmetric space $K \backslash G$. A functions $f$ on $S$ is $K$-invariant if

Received by the editors January 4, 1990.

1980 Mathematics Subject Classification (1985 Revision). Primary 22E30, 33A75, 43A85, 62H10; Secondary 20G20, 32A07, 44A10, 62E15.

This work was supported by National Science Foundation Grants DMS-8802929 and DMS-8807889. 
$f\left(k^{-1} s k\right)=f(s)$ for all $s \in S$ and $k \in K$.

An $n$-tuple $m=\left(m_{1}, \ldots, m_{n}\right)$ of nonnegative integers such that $m_{1} \geq \cdots \geq m_{n}$ is called a partition. Set $|m|=m_{1}+\cdots+m_{n}$. As a $G$-module the algebra of polynomial functions on $S$ is multiplicity free, the irreducible components are indexed by the partitions $m$, and in the $m$ th irreducible component is a $K$-invariant polynomial $Z_{m}$ on $S$, homogeneous of degree $|m|$ and unique up to scalar multiples, called a zonal polynomial. The zonal polynomials are normalized by the condition $(\operatorname{tr} s)^{d}=\sum_{|m|=d} Z_{m}(s)$. In the terminology of harmonic analysis, the zonal polynomials are polynomial spherical functions for the symmetric space $K \backslash G$.

Let $a_{1}, \ldots, a_{p}$ and $b_{1}, \ldots, b_{q}$ be complex parameters. From $[6,(6.1 .1)]$, the hypergeometric function ${ }_{p} F_{q}$ of matrix argument is a $K$-invariant function defined on $S$ by

$$
{ }_{p} F_{q}\left(a_{1}, \ldots, a_{p} ; b_{1}, \ldots, b_{q} ; s\right)=\sum_{m} \frac{\left[a_{1}\right]_{m} \cdots\left[a_{p}\right]_{m}}{\left[b_{1}\right]_{m} \cdots\left[b_{q}\right]_{m}} \frac{Z_{m}(s)}{|m| !}
$$

where $[a]_{m}=\prod_{i=1}^{n}\left(a-\frac{1}{2}(i-1) \nu\right)_{m_{i}}$ is the generalized truncated factorial for the matrix space $[6 ;(5.7 .2)],(a)_{k}$ denotes the ordinary truncated factorial, and $\nu=\operatorname{dim}_{\mathbf{R}} \mathbf{F}$. In analogy to the classical case, ${ }_{0} F_{0}(s)=e^{\text {tr } s}$ and ${ }_{1} F_{0}(a ;-; s)=\Delta(1-s)^{-a}$.

Hypergeometric functions have also been defined along the above lines on domains of positivity, a context which, in addition to the matrix spaces, also includes the Minkowski spaces (i.e., $\mathbf{R}^{n+1}$ with signature $\left.(n, 1)\right)$ and a certain exceptional $3 \times 3$ matrix space over the Cayley algebra $[4,10]$.

For the remainder of this note we set $\mathbf{F}=\mathbf{C}$, in which case $G=G L(n, \mathbf{C})$ is the complex $n \times n$ general linear group and $K=U(n)$ the unitary group. ${ }^{1}$ Since a Hermitian matrix $s$ can be diagonalized by an element of $K$, we can view the hypergeometric function (1) as a symmetric function of the eigenvalues $s_{1}, \ldots, s_{n}$ of the matrix $s$. Also, since the full matrix space $\mathbf{C}^{n \times n}$ is the complexification of the real space $S$, by analytic continuation we can view (1) as defining the hypergeometric function on $\mathbf{C}^{n \times n}$.

\footnotetext{
${ }^{1}$ Only for the case of complex matrix space, among all the domains of positivity, does the group $G$ have complex structure relative to which the maximal compact subgroup $K$ is a real form of $G$. This property over the complex field is the crucial structure upon which the detailed results of this paper ultimately rest.
} 


\section{WEYL'S CHARACTER FORMULA}

The partitions $m$ that index the zonal polynomials also parametrize the irreducible finite-dimensional (complex) polynomial representations $\lambda=\lambda_{m}$ of $G$. Set $\chi_{m}(s)=\operatorname{tr}\left(\lambda_{m}(s)\right)$. Then, over the complex field, the zonal polynomials are the normalized characters

$$
Z_{m}(s)=\omega_{m} \chi_{m}(s)
$$

of representations of $G$. By Weyl's character formula [12]

$$
\chi_{m}(s)=\frac{\operatorname{det}\left(s_{i}^{m_{j}+n-j}\right)}{V(s)}
$$

where $V(s)=V\left(s_{1}, \ldots, s_{n}\right)=\prod_{1 \leq i<j \leq n}\left(s_{i}-s_{j}\right)$ is the Vandermonde determinant. The constant $\omega_{m}$ is available from the literature $[9,10]$, but our methods provide a direct proof that

$$
\omega_{m}=\frac{|m| ! d_{m}}{[n]_{m}}
$$

where $d_{m}$ is the degree of $\lambda_{m}$. Formulas (2)-(4), together with a generalization of (1) involving two matrix arguments, lead to the following crucial result.

\section{EXPLICIT EVALUATION OF THE HYPERGEOMETRIC FUNCTIONS}

Let ${ }_{p} \mathscr{F}_{q}$ denote the classical hypergeometric function (i.e., the case $n=1)$. Then

$$
\begin{aligned}
{ }_{p} F_{q} & \left(a_{1}, \ldots, a_{p} ; b_{1}, \ldots, b_{q} ; s\right) \\
\quad= & \frac{\operatorname{det}\left(s_{i}^{n-j}{ }_{p} \mathscr{F}_{q}\left(a_{1}-j+1, \ldots, a_{p}-j+1 ; b_{1}-j+1, \ldots, b_{q}-j+1 ; s_{i}\right)\right)}{V(s)}
\end{aligned}
$$

Formula (5) allows one to transfer known properties of the classical hypergeometric functions to the matrix argument counterparts. Examples include precise asymptotic information and systems of differential equations, of which the following are representative samples.

\section{ASYMPTOTIC FORMULA AS $s_{i} \rightarrow \infty$ FOR $i=1, \ldots, n$}

Suppose $p \leq q$ and the series (1) does not terminate. Let $\beta=q+1-p, a=\sum_{i=1}^{p} a_{i}$, and $b=\sum_{i=1}^{q} b_{i}$. Then 
(6)

$$
\begin{array}{r}
\prod_{i<j}\left(\frac{s_{i}^{1 / \beta}-s_{j}^{1 / \beta}}{s_{i}-s_{j}}\right)^{-1}{ }_{p} F_{q}\left(a_{1}, \ldots, a_{p} ; b_{1}, \ldots, b_{q} ; s\right) \\
\sim \frac{\left(2 \pi^{n}\right)^{(1-\beta) n / 2}}{\beta^{n / 2}} \frac{\prod_{i=1}^{q} \Gamma_{n}\left(b_{i}\right)}{\prod_{i=1}^{p} \Gamma_{n}\left(a_{i}\right)}(\operatorname{det} s)^{[(\beta-1)(n-1)+a-b] / \beta} \\
\times \exp \left(\beta \sum_{i=1}^{n} s_{i}^{1 / \beta}\right) .
\end{array}
$$

Moreover, the error term is $O\left(\sum_{i=1}^{n} s_{i}^{-1 / \beta}\right)$.

In (6), and throughout, $\Gamma_{n}(a)=\pi^{n(n-1) / 2} \prod_{i=1}^{n} \Gamma\left(a+m_{i}-(i-1)\right)$ is the (generalized) gamma function for the cone $P[6 ;(5.6 .1)]$.

\section{Differential equations}

For any subset $A$ of $\{1, \ldots, n\}$, define the differential operators

(7) $\mathfrak{D}_{A}=\left[\prod_{k \in A} \partial_{k} \prod_{i=1}^{q}\left(\partial_{k}+b_{i}-n\right)\right]\left[\prod_{k \notin A} s_{k} \prod_{i=1}^{p}\left(\partial_{k}+a_{i}-n+1\right)\right]$ where $\partial_{k}=s_{k} \partial / \partial s_{k}$, and set

$$
\mathfrak{L}_{j}=V(s)^{-1}\left[\sum_{|A|=j} \mathfrak{D}_{A}-\left(\begin{array}{c}
n \\
j
\end{array}\right) \mathfrak{D}_{\phi}\right] V(s)
$$

for $j=1, \ldots, n$ (where $\phi$ denotes the empty set). Thensubject to conditions of symmetry, analyticity, and normalization at the origin - the hypergeometric function (1) is the unique solution to the system $\mathfrak{L}_{j} F=0$ of $n$ partial differential equations.

Note that the formulas in the previous two paragraphs reduce to well known classical results when $n=1$. We remark that formula (5) also yields new information about classical hypergeometric functions through use of the matrix-argument versions $[7,11]$.

\section{OPERATOR-VALUED HYPERGEOMETRIC FUNCTIONS ON $\mathbf{C}^{n \times n}$}

Let $\Delta$ denote the determinant and $d_{*} r$ be $G$-invariant measure on $P$. Fix a partition $m$ and the associated representation $\lambda=\lambda_{m}$ of $G$ acting in a Hilbert space $\mathscr{V}$, let $\mathscr{L}(\mathscr{V})$ be the space of linear transformations on $\mathscr{V}$, and define a family of hypergeometric functions on $\mathbf{C}^{n \times n}$ with values in $\mathscr{L}(\mathscr{V})$, starting with

$$
{ }_{0} F_{0}(\lambda \mid z)=e^{\operatorname{tr} z} I
$$


where $I$ is the identity on $\mathscr{V}$. For larger values of $p$ and $q$, we employ inductively the definitions

$$
\begin{aligned}
& \Delta(z)^{-a_{p+1}} \lambda(z)^{-1}{ }_{p+1} F_{q}\left(\lambda \mid a_{1}, \ldots, a_{p+1} ; b_{1}, \ldots, b_{q} ; z^{-1}\right) \\
& \quad=\frac{1}{\Gamma_{n}\left(a_{p+1}\right)} \int_{P} e^{-\operatorname{tr} z r} \Delta(r)^{a_{p+1}} \lambda(r)_{p} F_{q}\left(\lambda \mid a_{1}, \ldots, a_{p} ; b_{1}, \ldots, b_{q} ; r\right) d_{*} r
\end{aligned}
$$

and

$$
\begin{aligned}
& \Delta(z)^{-b_{q+1}} \lambda(z)^{-1}{ }_{p} F_{q}\left(\lambda \mid a_{1}, \ldots, a_{p} ; b_{1}, \ldots, b_{q} ; z^{-1}\right) \\
& \quad=\frac{1}{\Gamma_{n}\left(b_{q+1}\right)} \int_{P} e^{-\operatorname{tr} z r} \Delta(r)^{b_{q+1}} \lambda(r)_{p} F_{q+1}\left(\lambda \mid a_{1}, \ldots, a_{p} ; b_{1}, \ldots, b_{q+1} ; r\right) d_{*} r .
\end{aligned}
$$

We call ${ }_{p} F_{q}\left(\lambda \mid a_{1}, \ldots, a_{p} ; b_{1}, \ldots, b_{q} ; z\right)$ the operator-valued hypergeometric function on $\mathbf{C}^{n \times n}$ of weight $\lambda$. Note the covariance property

$$
\begin{aligned}
& { }_{p} F_{q}\left(\lambda \mid a_{1}, \ldots, a_{p} ; b_{1}, \ldots, b_{q} ; g z g^{-1}\right) \\
& \quad=\lambda(g)_{p} F_{q}\left(\lambda \mid a_{1}, \ldots, a_{p} ; b_{1}, \ldots, b_{q} ; z\right) \lambda(g)^{-1}
\end{aligned}
$$

for $g \in G$.

We can establish analogues for operator-valued hypergeometric functions of matrix argument of familiar classical formulas. For example, the operator-valued binomial theorem ${ }_{1} F_{0}(\lambda \mid a ;-; z)=$ $[a]_{m} \Delta(1-z)^{-a} \lambda(1-z)^{-1}$ holds for $\operatorname{Re} z>1$. The operator-valued Bessel function, defined in [5; (4.1)] by an integral over $K$, is given by $J_{\lambda}\left(2 r^{1 / 2}\right)=\pi^{n(n-1) / 2} \lambda\left(r^{1 / 2}\right)_{0} F_{1}(\lambda \mid-; n ;-r)$ for $r \in P$. The operator-valued confluent hypergeometric function has an Euler integral representation

$$
\begin{aligned}
& { }_{1} F_{1}(\lambda \mid a ; b ; z) \\
& \quad=\frac{\Gamma_{n}(b)}{\Gamma_{n}(a) \Gamma_{n}(b-a)} \int_{0<r<1} e^{\operatorname{tr} z r} \Delta(r)^{a} \Delta(1-r)^{b-a-n} \lambda(r) d_{*} r
\end{aligned}
$$

for all $z \in \mathbf{C}^{n \times n}$, valid for $\operatorname{Re} a>n-m_{n}-1$ and $\operatorname{Re}(b-a)>n-1$; and the operator-valued Gaussian hypergeometric function has an Euler integral representation

$$
\begin{aligned}
& { }_{2} F_{1}(\lambda \mid a, b ; c ; z) \\
& =\frac{\Gamma_{n}(c)}{\Gamma_{n}(b) \Gamma_{n}(c-b)}[a]_{m} \int_{0<r<1} \Delta(r)^{b} \Delta(1-r)^{c-b-n} \Delta(1-z r)^{-a} \lambda(r) \lambda(1-z r)^{-1} d_{*} r
\end{aligned}
$$


for $\operatorname{Re} z<1$, which holds when $\operatorname{Re} b>n-m_{n}-1$ and $\operatorname{Re}(c-b)>$ $n-1$.

\section{OPERATOR-VALUED DIFFERENTIATION}

By applying differential operators to the scalar-valued hypergeometric functions, we obtain the analytic continuation of the operator-valued hypergeometric functions. Specifically, if $\partial / \partial z=$ $\left(\partial / \partial z_{i j}\right)$, then

$$
\begin{aligned}
& { }_{p} F_{q}\left(\lambda \mid a_{1}, \ldots, a_{p} ; b_{1}, \ldots, b_{q} ; z\right) \\
& \quad=\lambda(\partial / \partial z)_{p} F_{q}\left(a_{1}, \ldots, a_{p} ; b_{1}, \ldots, b_{q} ; z\right) .
\end{aligned}
$$

\section{ADDITION FORMULAS}

For $z, w$, and $z+w$ in the domain of the hypergeometric function,

$$
\begin{aligned}
& { }_{p} F_{q}\left(a_{1}, \ldots, a_{p} ; b_{1}, \ldots, b_{q} ; z+w\right) \\
& \quad=\sum_{m} \frac{\omega_{m}}{|m| !} \operatorname{tr}\left(\lambda_{m}(z)_{p} F_{q}\left(\lambda_{m} \mid a_{1}, \ldots, a_{p} ; b_{1}, \ldots, b_{q} ; w\right)\right) .
\end{aligned}
$$

The analogous multiplication formula

$$
\begin{aligned}
& { }_{p} F_{q}\left(a_{1}, \ldots, a_{p} ; b_{1}, \ldots, b_{q} ; z w\right) \\
& \quad=\sum_{m} \frac{\omega_{m}}{|m| !} \frac{\left[a_{1}\right]_{m} \cdots\left[a_{p}\right]_{m}}{\left[b_{1}\right]_{m} \cdots\left[b_{q}\right]_{m}} \operatorname{tr}\left(\lambda_{m}(z) \lambda_{m}(w)\right)
\end{aligned}
$$

is rather trivial.

\section{CONCLUDING REMARKS}

The above results-together with generalizations of other aspects of the classical theory, such as summation theorems (e.g., Gauss' and Saalschutz' formulas, Kummer's and Thomae's transformations, etc.), orthogonal polynomials, Mellin-Barnes representations, differentiation properties, and contiguous relationswill be treated in full detail in [8]. A second paper, jointly with H. Ding, will develop a number of the new constructs-e.g., the operator-valued hypergeometric functions and differential operators-for all domains of positivity. In other related work, [3] applies the operator-valued Bessel functions on domains of positivity to representation theory of the automorphism groups of Hermitian symmetric spaces of tube type, and [1] and [2] initiate the study of operator-valued hypergeometric functions on Siegel domains of type II. 


\section{REFERENCES}

1. H. Ding, Operator-valued Bessel functions on Schrödinger-Fock spaces and Siegel domains of type. II, preprint.

2. __ Operator-valued Bessel functions on Schrödinger-Fock spaces and Siegel domains of type II: holomorphic discrete series and harmonic representations (in preparation).

3. H. Ding and K. I. Gross, Operator-valued Bessel functions on symmetric cones and holomorphic discrete series (in preparation).

4. J. Faraut and A. Korányi, Fonctions hypergéometriques associées aux cônes symétriques, C. R. Acad. Sci. Paris Sér. I 307 (1988), 555-558.

5. K. I. Gross and R. A. Kunze, Generalized Fourier-Bessel transforms and holomorphic discrete series, Proc. Internat. Conf. in Harmonic Analysis, Lecture Notes in Math., vol. 261, Springer-Verlag, Berlin and New York, 1973, pp. 79-122.

6. K. I. Gross and D. St. P. Richards, Special functions of matrix argument, I: Algebraic induction, zonal polynomials, and hypergeometric functions, Trans. Amer. Math. Soc. 301 (1987), 781-811.

7. __ Total positivity, spherical series, and hypergeometric functions of matrix argument, J. Approximation Theory 59 (1989), 224-246.

8. __ Special functions of matrix argument, II: The fine structure of hypergeometric functions on complex matrix space (in preparation).

9. A. T. James, Distributions of matrix variates and latent roots derived from normal samples, Ann. of Math. Statistics 35 (1964), 475-501.

10. A. Korányi, Hua-type integrals, hypergeometric functions, and symmetric polynomials, preprint.

11. D. St. P. Richards, Totally positive kernels, Polya frequency functions, and generalized hypergeometric series, Linear Algebra Appl. 137/138 (1990), 467-478.

12. H. Weyl, The classical groups, Princeton Univ. Press, Princeton, NJ, 1939.

\footnotetext{
Department of Mathematics and Statistics, University of Vermont, BURLINGTON, VERMONT 05405

Department of Mathematics, University of Virginia, Charlottesville, VIRGINIA 22903
} 
